\title{
Pre and Post examination evaluation of MCQs and SEQs Items in Undergraduate MBBS mock examination.
}

1. MBBS, M.Phil, Ph.D, MHPE, MBA Professor Biochemistry

Shalamar Medical and Dental College,

University of Health Sciences, Lahore.

2. MBBS, FCPS, DCPS, MCPS

Professor Obstetrics \& Gynecology Fatima Jinnah Medical University.

Correspondence Address:

Prof. Dr. Anila Jaleel

Department of Biochemistry Shalamar Medical and Dental College University of Health Sciences, Lahore. aneelajaleel@hotmal.com

Article received on: 09/01/2020

Accepted for publication: $11 / 05 / 2020$

\section{Anila Jaleel ${ }^{1}$, Zohra Khanum ${ }^{2}$}

ABSTRACT... Objectives: To evaluate the quality of MCQs and SEQs pre and post Mock examination of physiology and biochemistry and correlation between the scores of both at private medical college Lahore. Study Design: Descriptive study and convenient sampling was done. Setting: Fatima Memorial College of Medicine and Dentistry, Lahore. Period: September 2016 to September 2017. Material \& Methods: 149 students in Physiology and 143 in biochemistry took Mock examination. $45 \mathrm{MCQs}$ and 9 SEQs each in biochemistry and physiology were prepared according to the table of specification provided by University of Health Sciences (UHS) Lahore, Pakistan. Item assessment according to Blooms taxonomy was done and item flaws identified with cover test done for structural validity of the paper before the exam by two medical educationists. Item analysis with difficulty index, discrimination index and distraction efficiency were done post examination. Results: $84.4 \%$ in physiology and $51.1 \%$ in biochemistry were of recall type (C1 level according to Blooms taxonomy), and 58\% SEQs in physiology and $50 \%$ in biochemistry were $\mathrm{C} 1.20 \%$ and $28 \%$ MCQs in physiology and biochemistry respectively pass cover test and were without item writing flaws. Difficulty index shows that $53.3 \%$ in physiology and $48.8 \%$ in biochemistry needs modifications. $48.8 \%$ of MCQs in physiology and $15.5 \%$ in biochemistry need modification in discriminatory index. Similarly $59.5 \%$ and $64.0 \%$ of MCQs had functional distractors in physiology and biochemistry respectively. Conclusion: The study concluded that Mock examination in subjects of physiology and biochemistry had item writing flaws, more MCQs were of $\mathrm{C} 1$ level and showed majority items in good difficulty, discriminatory index with two third functional distractors were present

Key words: Cover Test, Difficulty Index, Discriminatory Index, Distractor Effectiveness, Mock Examination.

Article Citation: Jaleel A, Khanum Z. Pre and Post examination evaluation of MCQs and SEQs Items in Undergraduate MBBS mock examination. Professional Med J 2020; 27(12):2749-2754. https://doi.org/10.29309/TPMJ/2020.27.12.4474

\section{INTRODUCTION}

Assessment is an important part of learning cycle and is used both as formative and summative. Formative assessment helps not only teachers to identify the gap in learning of students but also to modify the teaching strategies. ${ }^{1}$ Summative assessment is done either at end of year or at end of semester in most of the medical schools in Pakistan. The tools of assessment are mostly MCQs, SEQs, SAQs, OSPE, OSCE and Viva Voce. However in clinical assessments long and short cases are also used for bed side assessments. The MCQs and SEQs are part of the written examination at University of Health Sciences (UHS) Lahore. Mock examination carried out before the professional examinations are done at pattern of final examinations and students who failed to pass the mock are not allowed to take professional examinations. Due to high stake of this examination, the quality of MCQs and SEQs matters a lot. A number of medical colleges have assessment units which ensure the quality of the items in MCQs and questions in SEQs. Moreover post examination evaluation of assessment i.e. difficulty index, discriminatory index and distraction factor help in improving the quality of assessments in the next examinations. ${ }^{2}$ Studies by Patel and Mohajen (2013), Mehta and Mohsin (2014), Baig et al. (2014) and Mahmood (2015) indicate the psychometric analysis of items done in various undergraduate MBBS subjects. ${ }^{1,2,3,4}$ 
The study was done to evaluate the quality of MCQs and SEQS in physiology and biochemistry and correlation between the scores of them in Mock examination at private medical college of Lahore.

\section{OBJECTIVES}

Is to evaluate the quality of MCQs and SEQS pre and post Mock examination of physiology and biochemistry and correlation between scores of two modalities at private medical college of Lahore

\section{MATERIAL \& METHODS}

Study was conducted at Fatima Memorial College of Medicine and Dentistry, Lahore from 2016 to 2017. It was a descriptive study and convenient sampling was done. Informed consent was obtained from students of second year MBBS and the study was approved by Institutional review board (IRB) of FMH and UHS. Included were second year MBBS students and MCQs and SEQs papers of biochemistry and physiology were analyzed. Students of third, fourth and final years MBBS were excluded from the study and OSPE and Viva were not considered for analysis.

\section{Study Protocol}

Out of 150 second year MBBS students, 149 took the written Mock examination of physiology and 143 students in biochemistry comprising of one MCQ and one SEQ paper in each subject.

Each paper consist of 45 MCQs and 9 SEQS according to the table of specification of the University (UHS) Lahore, Pakistan. Final scores of SEQS were the mean of scores of each SEQ by two independent examiners.

Item analysis with difficulty index, discrimination index and distraction factor were done post examination. Standard setting was done at $50 \%$ or 45/90 using absolute method. Scores of MCQs and SEQs were correlated using Pearson's method.

Data analysis was done using SPSS 21. Difficulty, discriminatory index and distractors efficacy were calculated by the standard formulas (Gajjar et al.,
2014; Mehta and Mokhasi, 2014).

\section{RESULTS}

Total of 90 MCQs and 18 SEQs were reviewed in Basic Sciences i.e physiology and biochemistry. Figure-1 shows $84.4 \%$ in physiology and $51.1 \%$ in biochemistry were of recall of knowledge, while $13.3 \%$ and $28.8 \%$ of interpretation level and $2.22 \%$ and $20 \%$ of problem solving in physiology and biochemistry respectively. Out of 9 SEQs in each subject $58.8 \%$ and $50 \%$ of SEQs were of recall of knowledge, $17.6 \%$ and $27.7 \%$ interpretation level and $23.5 \%$ and $22.2 \%$ were of problem solving in physiology and biochemistry respectively.

Figure-2 shows the item writing flaws in MCQs in both subjects and ability to pass cover test. Passing the cover test was rated as $1.20 \%$ and $28 \%$ were rated 1 and $80 \%$ and $71.2 \%$ had one or more item writing flaws in physiology and biochemistry respectively.

Figure-3 shows difficulty, discriminatory index and distractor effectiveness. $62.2 \%$ of MCQs out 45 were in good and acceptable range of difficulty index while $53.3 \%$ need review in physiology while $51 \%$ of MCQs in biochemistry were good and acceptable while (48.8\%) need to be reviewed. Similarly $53.3 \%$ of MCQs in physiology and $84.3 \%$ in biochemistry were either excellent, good or acceptable of discriminatory index while $4.8 \%$ and $15.5 \%$ in physiology and biochemistry need to be reviewed.

Distractor effectiveness of options in MCQs $>5$ were $59.5 \%$ in physiology and $64 \%$ in biochemistry, while $40.4 \%$ of physiology and $36 \%$ in biochemistry had distractor effectiveness $<5$ i.e. were nonfunctional distractors (Figure-3).

Correlation of scores of MCQs and SEQs was determined by Pearson's correlation, which was significant (0.049) in physiology and highly significant i.e. 0.001 in biochemistry (Figure-4). 


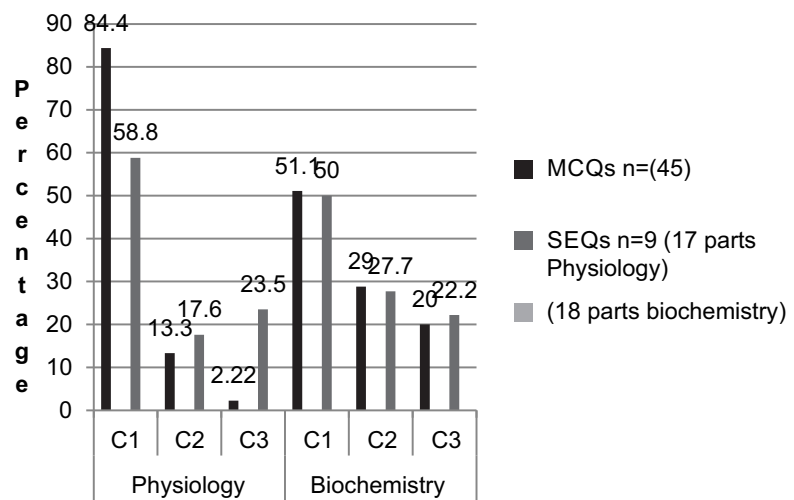

Figure-1. Knowledge Level of Items Based on Bloom's Taxonomy

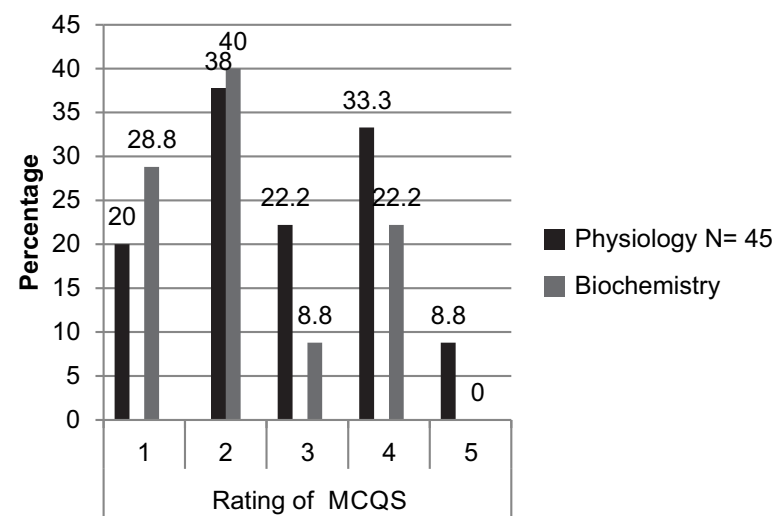

Figure-2. Rating of MCQs in physiology and biochemistry

\begin{tabular}{|c|l|}
\hline Rating & \multicolumn{1}{|c|}{ Specifications } \\
\hline 1. & Cover test pass with no item-writing flaws \\
\hline 2. & Cover test pass with 1 to 2 item-writing flaws \\
\hline 3. & Dubious cover test dubious with no item writing flaws \\
\hline 4. & Cover test fail with 1 to 2 item-writing flaws \\
\hline 5. & Cover test fail with more than 2 item-writing flaws \\
\hline (Palmer and Devitt, $2007 ;$ Bloom et al., 1956$)$ \\
\hline
\end{tabular}

(Palmer and Devitt, 2007; Bloom et al., 1956)
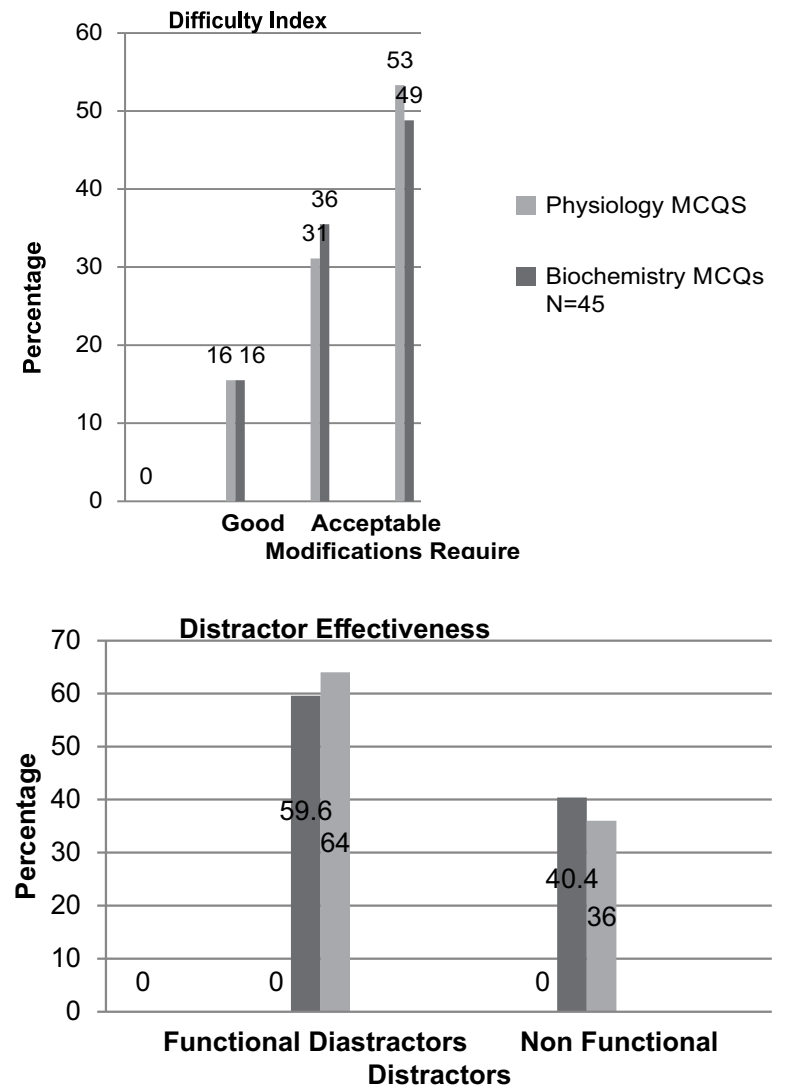

Figure-3. Difficulty index discriminatory index and distractor effectiveness of MCQS in physiology and biochemistry
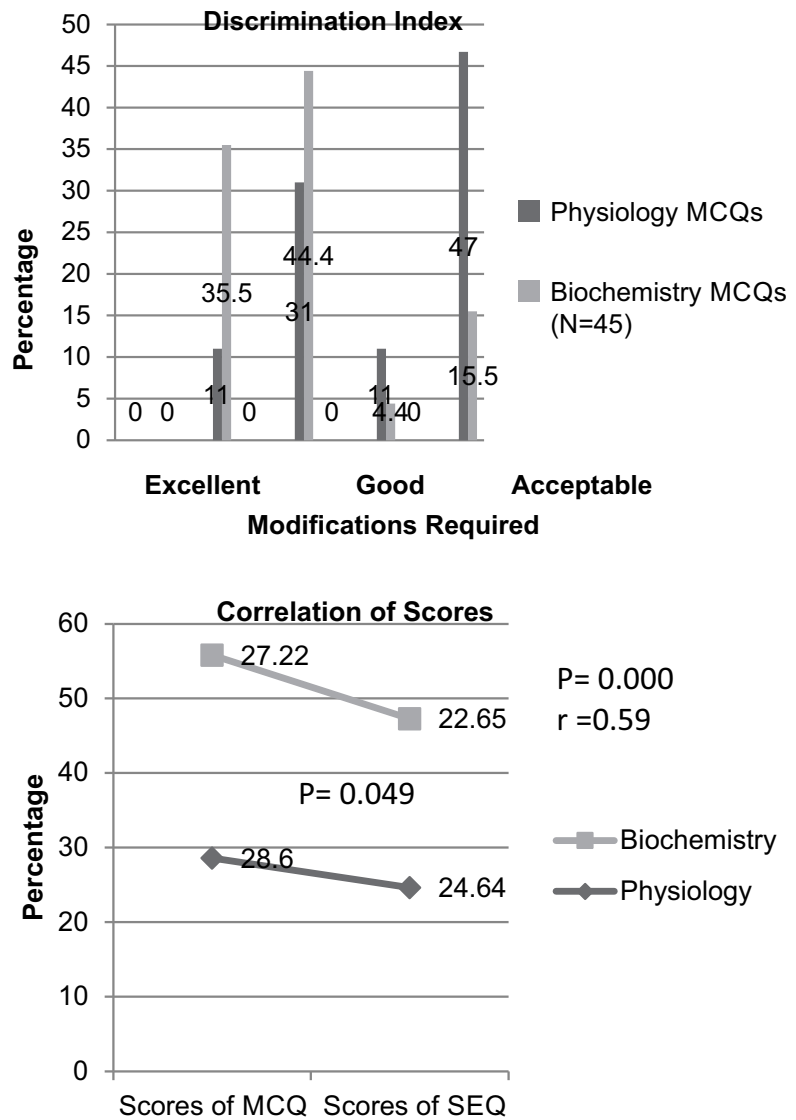

Figure-4. Correlation of MCQ and SEQ scores in subjects of physiology and biochemistry 


\section{DISCUSSION}

Assessment plays key role in learning, whether it is formative or summative. Selection of appropriate tool for assessment is the key to gage the learning of students. Summative assessment is assessed at University of Health Sciences (UHS) by written and viva voce and practical examinations, which each student has to pass separately. Mock examinations are conducted by each medical college affiliated with UHS before professional examinations. Each student has to pass the examination for being eligible to take professional examination.

The written Mock examination of a private medical college was assessed in this study for quality of MCQs and SEQs. One best type MCQs and SEQs were assessed for difficulty index, discriminatory index, distractor effectiveness, ability to pass cover test and correlation between MCQs and SEQs. Most of the MCQs (84.4\% in physiology and $51.1 \%$ in biochemistry) were of recall type with few of interpretation and problem solving level with only $20 \%$ and $28.8 \%$ pass the cover test without any item writing flaws (IWF) respectively. Similarly 58\% and 59\% SEQs in physiology and biochemistry respectively were of recall type. Though it is difficult to construct C3 level items in basic sciences but little more effort by the faculty may increase such items especially in $21^{\text {st }}$ century when the medical sciences are being taught contextually and with relevance. IWFs can be minimized by proof reading the items by subject specialist and medical educationist before finalizing the examination paper. Difficulty index showed 53.3\% MCQs in physiology and $48.8 \%$ in biochemistry needs modifications. Similar pattern was seen in discriminatory index with $48 \%$ and $15.5 \%$ required modifications in both subjects respectively. $40.4 \%$ and $36 \%$ were nonfunctional distractors respectively. MCQs and SEQs correlated positively and significantly in two subjects.

Several studies support the data in our study. Study done at Qassim University (KSA) to examine the undergraduate students in medicine showed that $40 \%$ MEQS and $60 \%$ MCQs were of C3 type, while others assess comprehension and recall. ${ }^{5}$

Another study done in Ophthalmology showed $30 \%$ MCQs and 10\% SEQs of interpretation level. ${ }^{4}$ Another study conducted to examine baccalaureate nursing programs from 20056 showed $53.8 \%$ of MCQs with no item writing flaws while $46.2 \%$ with one or more item writing flaws. C1 type MCQs were $91.1 \%$ while only $4.7 \%$ were of C2 and C3 type. ${ }^{6} 35.1 \%$ were nonfunctional distractors. Another study showed $38 \%$ nonfunctional distractors out of 477 items. $^{7}$

Similar study showed $76 \%$ and $24 \%$ of MCQs at $\mathrm{C} 1$ and $\mathrm{C} 2$ levels respectively. 69 IWFs were found in 150 MCQs with $30.43 \%$ implausible distracters. $^{3}$ Studies by Downing (2005) and Elisworth et al., (1990) showed similar results for IWF in examinations. ${ }^{9}$ Study by Mehta and Mohsin, (2014) showed $62 \%$ items within acceptable range and $38 \%$ require modifications. Another study showed $61 \%$ items in acceptable to excellent range of difficulty index while $24 \%$ items were easy and $15 \%$ difficult. Similar pattern was shown by study by Patel and Mohajen, (2013). Study by Hingorjo and Jaleel, (2012) showed 78\% items were of average difficulty with 81.41 distractor efficiency in physiology examination. ${ }^{10}$ Studies done by Mehta and Mokhasi (2014) showed only $30 \%$ items of poor discriminatory index, while $35.3 \%$ were nonfunctional distractors, while study by Mahjabeen et al., (2017) also showed similar results. ${ }^{11,12}$ Study conducted by Mawlood et al at., anlaysed 180 single best questions in Paediatric dentistry department postgraduate examinations and identified $81 \%$ as recall and $19 \%$ of higher order thinking. ${ }^{13}$ Two studies were conducted by Hassan et al., in 2016 and 17 to determine the item anlaysis and reliability of undergraduate examination in Malaysia and it impact. ${ }^{14,15}$ Several other studies were carried out in different medical schools in the world and have shown similar results. ${ }^{16-22}$

Vigorous training of basics and clinical faculty is required to construct MCQs and SEQs of interpretation and problem solving level. Although MCQs and SEQs development of C3 level is easy in clinical sciences compared to basic sciences 
but clinical relevance in basic sciences create a space for more problem solving type questions. Specified time must be allocated by the faculty in examination department to construct and proof read the papers pre and post examination.

The study helped us to identify the fault in construction of items, easiness and difficulty of the items and whether they were able to discriminate between good and poor students. These factors affect the validity and reliability of the examination. MCQs with negative discriminatory index affect the validity of the examination and should be removed from the bank. The regulation of PMDC for mandatory medical education department in each medical college with full time qualified medical educationist must be appreciated. Although faculty development workshops are being conducted on regular basis in quality medical colleges but hands on training of the faculty in Item construction with more time being spent on it would improve the quality of examinations in near future

\section{CONCLUSION}

The study concluded that Mock examination conducted at $\mathrm{FMH}$ college of Medicine and Dentistry in subjects of physiology and biochemistry had item writing flaws, more MCQs were of $\mathrm{C} 1$ level and showed majority items in good difficulty, discriminatory index with two third functional distractors were present

\section{LIMITATION OF THE STUDY}

The study was done only in second year MBBS written examination while quality of other modalities like OSPE and viva was not considered.

\section{CONFLICT OF INTEREST}

There is no conflict of interest regarding the study Copyright@ 11 May, 2020.

\section{REFERENCES}

1. Patel K, Mahajan N. Itemized analysis of questions of multiple choice question (MCQ) exam. IJSR Int J Sci Res, 2013; 2: 279-280.

2. Mehta G, Mokhasi V. Item analysis of multiple choice questions-an assessment of the assessment tool. Int J Health Sci Res. 2014; 4:197-202.
3. Baig M, Ali S K, Ali S, Huda N. Evaluation of multiple choice and short essay question items in basic medical sciences. Pakistan journal of medical sciences. 2014;, 30(1): 3-5.

4. Mahmood H. Correlation of MCQ and SEQ scores in written undergraduate ophthalmology assessment. J Coll Physicians Surg Pak. 2015 Mar 1;25(3):185-88.

5. Moeen-Uz-Zafar Khan, B. M. Evaluation of modified essay questions (MEQ) and multiple choice questions (MCQ) as a tool for assessing the cognitive skills of undergraduate medical students. International journal of health sciences. 2011; 5(1): 39-43.

6. Tarrant M, Ware, J. Impact of item-writing flaws in multiple-choice questions on student achievement in high-stakes nursing assessments. Medical education. 2008; 42(2): 198-206.

7. Haladyna $T$ M, Downing $S M$. How many options is enough for a multiple-choice test item? Educational and Psychological Measurement. 1993; 53: 999-1010.

8. Downing $S M$. The effects of violating standard item writing principles on tests and students: The consequences of using flawed test items on achievement examinations in medical education. Advances in health sciences education. 2005; 10(2): 133-143.

9. Ellsworth R A, Dunnell P, Duell, O K. Multiple-choice test items: What are textbook authors telling teachers? The Journal of Educational Research.1990; 83(5): 289293.

10. Hingorjo M R, Jaleel F. Analysis of one-best MCQs: The difficulty index, discrimination index and distractor efficiency. JPMA-Journal of the Pakistan Medical Association. 2012 62(2), 142-147.

11. Mehta G, Mokhasi V. Item analysis of multiple choice questions-an assessment of the assessment tool. Int J Health Sci Res. 2014; 4: 197-202.

12. Mahjabeen W,Alam $S$, Hassan $U$ et al., Difficulty index, discrimination index and distractor efficiency in multiple choice questions. Ann. Pak. Inst. Med. Sci. 2017:310-314.

13. Mawlood Kowash, lyad Hussein, Manal Al Halabi. evaluating the quality of multiple choice question in paediatric dentistry postgraduate examinations. Sultan Qaboos Univ Med J. 2019; 19(2): e135-e141.

14. Hassan S and Hod_Rafidiyah - Use of item analysis to improve the quality of single best answer multiple choice question in summative assessment of Undergraduate Medical Students in Malaysia. Education in Medicine Journal. 2017; 9: 33-43. 
15. Hassan S, Mohd Amin R, Mohd Amin, Rebuan H, Aung MMT. Item analysis, reliability statistics and standard error of measurement to improve the quality and impact of multiple choice questions in undergraduate medical education in faculty of medicine at UniSZA. Malaysian Journal of Public Health Medicine. 2016; 16(3):7-15.

16. Hassan S, Mohd Amin R, Mohd Amin Rebuan H, Aung MMT. Item analysis, reliability statistics and standard error of measurement to improve the quality and impact of multiple choice questions in undergraduate medical education in faculty of medicine at UniSZA. Malaysian Journal of Public Health Medicine. 2016; 16(3):7-15.

17. Mukherjee P, Lahiri SK. Analysis of multiple choice questions (MCQs): Item and test statistics from an assessment in a medical college of Kolkata, West Bengal. IOSR J Dental Med Sci. 2015; 14:47-52.

18. Menon AR, Kannambra PN. Item analysis to identify quality multiple choice questions. Nat $\mathrm{J}$ Lab Med. 2017; 6: 7-10.

19. Tariq S, Tariq S, Maqsood S, Jawed S, Baig M. Evaluation of cognitive levels and item writing flaws in medical pharmacology internal assessment examinations. Pak J Med Sci. 2017; 33:866-70.
20. Botelho MG, Lam O, Watt RM, Leung D, Kember D. Evaluation of peer-generated MCQs to assess and support learning in a problem-based learning programme. Eur J Dent Educ. 2018; 22:e358-63.

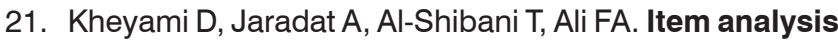
of multiple choice questions at the department of paediatrics, Arabian Gulf University, Manama, Bahrain. Sultan Qaboos Univ Med J 2018; 18:e68-74.

22. Field JC, Walmsley AD, Paganelli C, McLoughlin J, Szep S, Kavadella A, et al. The graduating European dentist: Contemporaneous methods of teaching, learning and assessment in dental undergraduate education. Eur J Dent Educ. 2017; 21:28-35.

23. Yodying Dangprapai, Popchai Ngamskulrungroj, Sansnee Senawong, Patompong Ungprasert, Azian Harun. development of a new scoring system to accurately estimate learning outcome achievements via single, best-answer, multiple-choice questions for preclinical students in a medical microbiology course. J Microbiol Biol Educ. 2020; 21(1): 21.1.4.

\section{AUTHORSHIP AND CONTRIBUTION DECLARATION}

\begin{tabular}{|c|l|l|c|}
\hline Sr. \# & \multicolumn{1}{|l|}{ Author(s) Full Name } & Contribution to the paper & Author(s) Signature \\
\hline 1 & Anila Jaleel & 1st Author & 2nd \\
2 & Zohra Khanum & 2nd Author \\
\hline
\end{tabular}

